\title{
HETEROGENEOUS CROWD SIMULATION
}

\author{
Srivastav R. Janapalli \\ Omar Hesham \\ Gabriel A. Wainer \\ Carleton University \\ Department of Systems and Computer Engineering \\ 1125 Colonel By Drive \\ Ottawa, ON, Canada \\ \{SrivastavJanapalli,Omar.Hesham\}@cmail.carleton.ca, gwainer@sce.carleton.ca
}

\begin{abstract}
Dense crowd simulations are being increasingly used for predicting congestion, planning protests and rallies, and threat assessment. It has been observed that human behavior is uncontrollable and often unpredictable; however, human motion is quite flexible over obstacles in their path and turn as per the need. These motions or the paths they take are majorly based on their individual personal space. Upon introducing these entities to a heterogeneous environment with vehicles, bikes, shopping carts and strollers, each with their own path and their own personal space, human motion drastically shift. Here we introduce a new set of models intended to study such behavior and try to mimic such situations.
\end{abstract}

Keywords: Crowd modelling, Centroidal Particles Dynamics, Heterogeneous Crowds.

\section{INTRODUCTION}

Like other physically based phenomena that exhibit complex interactions between entities and vehicles or objects in heterogeneous environment overtime, crowd motion can only be practically simulated by numerically calculated methods, unlike analytical solutions (Cao et al. 2009). Simulation studies have been in different fields, and numerous models have been built for understanding of crowds and their interaction with the surrounding environment (Andrade et al., 2006). For instance, crowd simulation algorithms have been widely used to generate plausible effects in games and computer animation (Lister and Day, 2012) as well as to predict the flow of pedestrians in urban environments and architectural models.

There is a substantial amount of research about the dynamics of such a compound system and wellestablished approaches can be distinguished in the study of pedestrian modelling, including cellular automata (Sarkar 2000, Joel 2012). In CA models we can see bottom-top approach, where each entity was able to apply simple principles approach, including synthetic walking rules and obstacle transitioning perception to simulate crowd behavior in densely populated scenarios (Ivancevic et al. 2010). In CA methods, the space is typically divided into a uniform grid, where every cell can either be available, occupied by an entity, or represent an obstacle. Every cell's future state is then determined based on the states of the cells in its local neighborhood. CA crowd models were rapidly developed and adopted, thanks to their parallel-friendly processing and inherent visualization (every cell is both the computational unit and the visual representation). Nevertheless, grid-based Eulerian evaluation of agent dynamics using discretized stepping and finite directions of motion does not faithfully reflect the fluidity of human motion trajectories. 
In order to deal with these issues, we proposed Centroidal Particle Dynamics (CPD), an explicit 2D model of the dynamics of response to violations of personal space. It is implemented through autonomous Lagrangian agents, which emergently recreate global phenomenon observed in dense crowds. Here, we expanded are interested in expanding our previous research including behavior observation in a heterogeneous environment including vehicles, bikes, and entities with their own path and their own personal space. We will discuss the model approach, and we will present multiple scenarios, each pertaining to different vehicle moment pattern and varying in crowd count i.e., crowd density change. We include flexibility for adding foreign objects and simulating traffic scenarios. We made use of some of such scenarios and reviewed in-depth analysis of different case studies. The simulation results show we can combine vehicles and pedestrians in a way that resembles real-life scenarios.

\section{BACKGROUND}

Replicating human decision-making is an overly ambitious endeavor, never mind simulating an entire crowd of them. To this end, the abstraction of motion dynamics by generalizing observed phenomenon is necessary to achieving a computable result. This section presents a brief overview of the multitude of methods developed to tackle this problem. Historically, the earliest crowd simulation methods were macroscopic in nature, simulating aggregate behavioral patterns, rather than actual individual trajectories in the scene. They were based on adapting existing fluid simulation models to incorporate aggregate human motion parameters. Later, with improved hardware capabilities and modeling methodologies, the ability to simulate individual entity-to-entity interactions have become computationally viable. For instance (Kullu et al. 2017) discusses the influence of shared information between subjects and how that effects crowd's behavior and movement. Similarly, the MENGA tool (Narain et al. 2009) has been used to simulate multiagents influencing crowds, discusses multiple ways of communication. By simulating multi-agents that influence crowd, communication between agents do not consider speech, language barriers or subtleties of human behaviors (resulting in a quasi-robotic behavior). In most cases the models are predefined with event types, confined environment, and fixed messages. Nevertheless, it is important to study situations where the agents are self-made, and new agents have no prior knowledge of scenario or environment to observe the change in their behavior.

In (Rangel et al. 2017) the authors discuss a time-based model to develop a model using entropy based on pedestrian time restraints. Entropy is associated with the decisions made by individuals and they compare the results with density changes. Entropy can drop to zero under densely crowded situations, leading to homogeneous behavior. Dynamic pedestrian crowds, instead, should consider adaptation to the situation and flow conditions. Likewise, it would be important considering more parameters like pedestrian stamina, mental and physical state, or even physically handicapped situations.

In (Hesham and Wainer 2017), we discussed various methods and models that are available. The Lagrangian-based method uses neighborhood detection; comparatively Eulerian method uses predefined and has direct accessibility (Zheng et al. 2016). In order to deal with these issues, we introduced the theory of Centroidal Particles where each agent knows their global path and uses personal space as boundaries while using directional vectors to set their route. An optimization technique is used to find the shortest trajectory. The idea is based on an American and French study on personal space in a normalized fashion and does not consider cultural or country variations in it (Pettré et al. 2009). The original research assumes 
that every entity is fully aware of his surroundings actively. Nevertheless, this is not the case, for example, when pedestrians walk using smartphones or listening to music.

The research in (Zheng et al. 2016) shows simulation on how humans react to various situations mainly navigation based on four main individual physiological treats. They conduct video analysis-based simulation to be more direct and objective and they use the RVO Library for simulation (Patil et al. 2011). Nevertheless, the RVO library parameters are limited and results in compromising decisions to assume two physiological characteristics into one parameter (Fabeković et al. 2007). Most simulations focus on behavioral patterns (Moussaid et al. 2011) and on understanding the human motions under stressful environments, such as evacuation procedures. (Huang et al. 2015) consider human rationality as a factor that impacts crowd evacuation model.

\section{EXTENDING CENTROIDAL PARTICLE MODELS}

As discussed earlier, our objective is to introduce heterogeneous crowd simulation, based on the Centroidal particle method. The CPD model has been built for simulation of homogeneous entities behavior analysis under variable density. The model includes the following components:

- CentroidalSofticles includes the main setup scene method. Uses the setup scene method and draws the canvas with a declared number of entities.

- Canvas includes the objects and knows how to render the entities linked with entities system and environment. It also has a private PGraphics surface and alters the entities destinations.

- Entity consists of setters and getters of entities to update the position vectors, create entity forces, updating entity positions and destination vectors.

- EntitySystem includes methods to define the entities initial positions, their direction of flow and destination.

- Mods has the ability to insert custom shapes and foreign entities to current existing crowds, making the scene heterogeneous. It has the core definitions of modifying forces. We can add obstacles from a PNG image and draw the scene obstacles from the methods defined in this file.

- RenderView consists of setters and getters of screen viewing properties.

- Shape loads the objects from the specified OBJ files defined by users, creates a softicle shapes and calculate the centroid position.

- Softicle consists setters and getters used by shape file for weight map and base shape.

The CPD engine only includes individual entities that are homogeneous. Each entity is represented as a 2D circle and they're respective personal apace is represented by another circle overlapping the entity with a predefined radius. We extended the method in order to allow polygonal objects like vehicles and shopping carts. To do so, the selected object is represented with a 3D cones around the objects/polygons to resemble their personal space. To represent this personal space as a 3D cone around the $2 \mathrm{D}$ object can be staged by Minkowski sum approach (Pustylnik and Sharir 2003). The idea is that, for given two sets P and Q, the sum $\mathrm{P} \oplus \mathrm{Q}$ (Equation 1) is constructed by considering a secondary polygon on multiple segments around each vertex of the primary polygon. The segments of the convolution will form a number of closed polygonal curves, generally represented as convolution circles. Based on this convolution circles of $\mathrm{P}$ and $\mathrm{Q}$ polygons, we compute the winding numbers which represent the faces formed by Minkowski boundary depicted in Figure 1 (Lien 2007). 


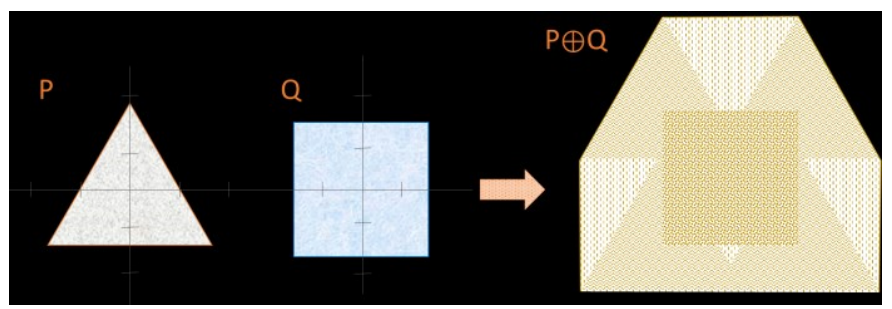

Figure 1. Minkowski sum representation of a triangle and a square.

We defined the Minkowski sum by considering individual vertices of a given 2D object like a circle and generating 3D structured cones on each vertex.
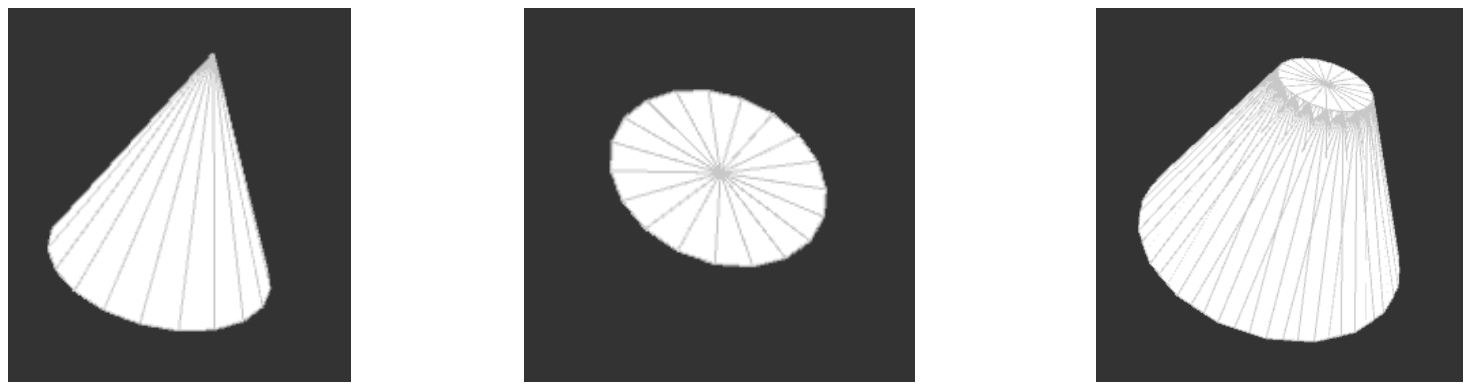

Figure 2. Representation of personal space of a disk with a cone through Minkowski sum. (a) cone with 30 vertices; (b) disk with 20 vertices; (c) Minkowski sum.

We generated a a Minkowski sum for a vehicle (in order to represent their personal space). First, we used an Autodesk Maya 2017 sample vehicle (obtained from 3dcad.com). After removing textures, we transformed the model into a mesh object to make it editable. Subsequently, we flattened out the object and drew another layer around the car. We implemented the Minkowski sum for all the vertices of the car model, representing the vehicle and the personal space of the car around it.
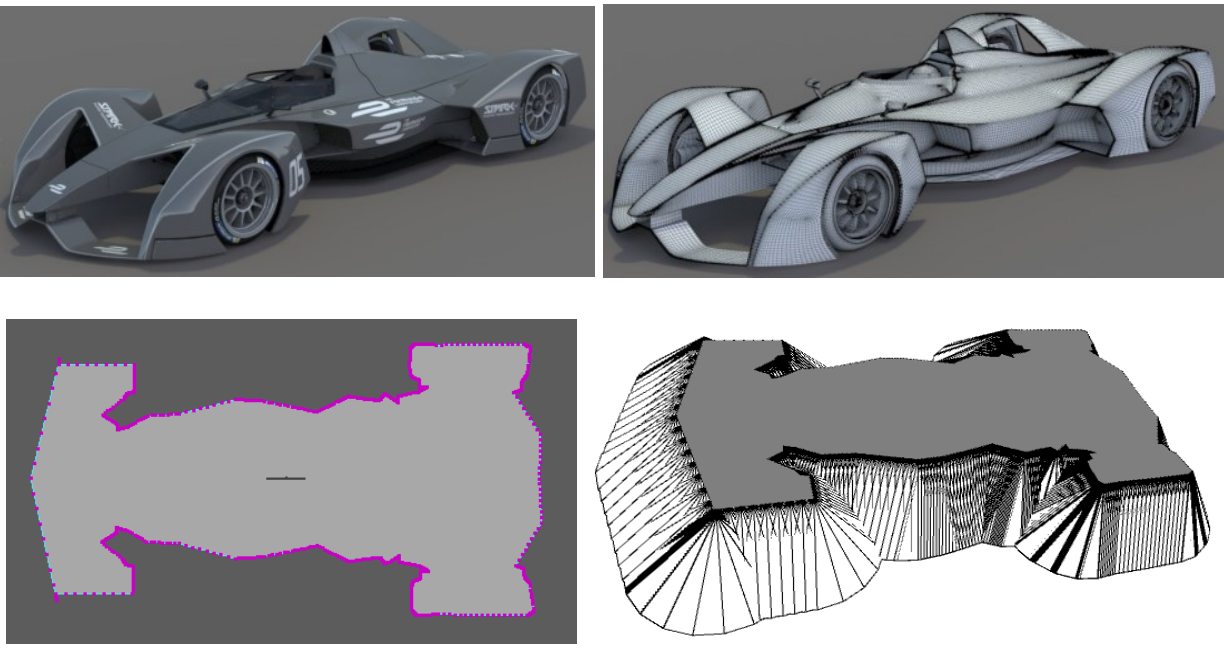

Figure 3. Converting a sample vehicle.

We followed this approach for other 3D models, and generated object files that can be used in our crowd simulation engine. 

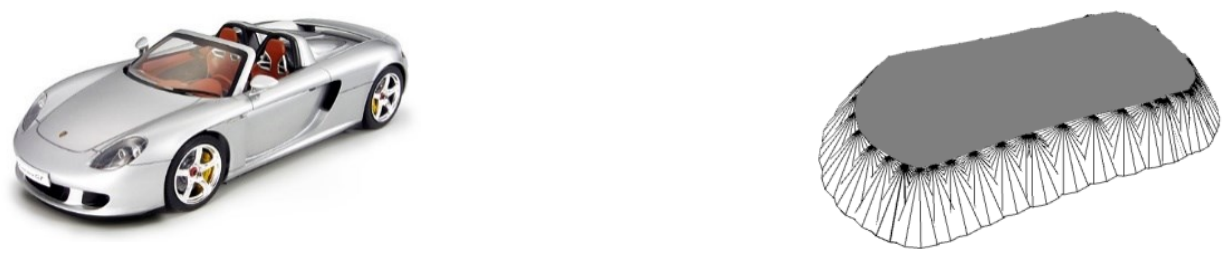

(a) Sports vehicle model.
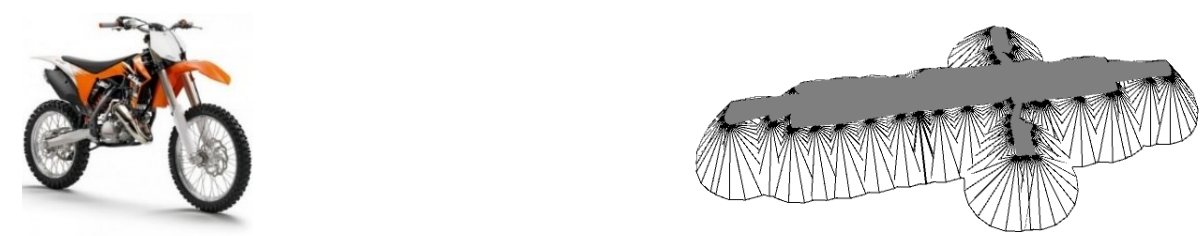

(b) Motorcycle model.
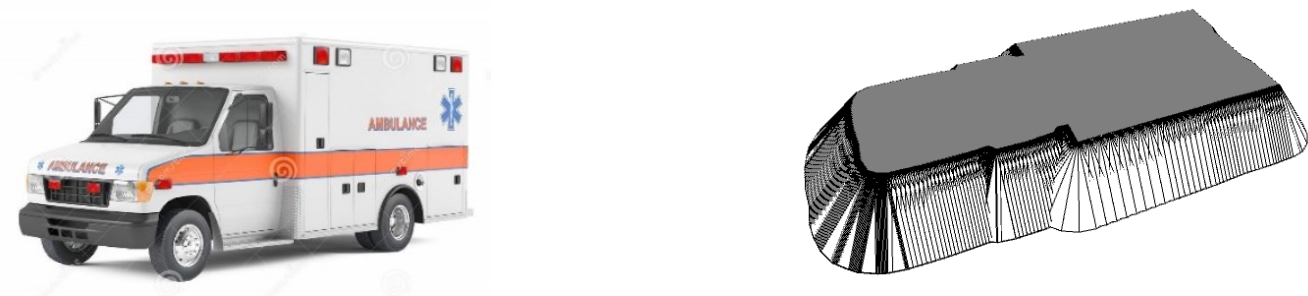

(c) Ambulance model.

Figure 4. Different models and approximation to work within the crowd engine.

These models needed to be included in the heterogeneous environment, which is different from the traditional homogeneous setup (where the entities are all humans represented as circles). With heterogeneity and n-sided polygon profiles, we need to compute the geometric center of their shape, in order to account for irregular shapes and to allow them to stand still when no external forces are experienced.

$$
\text { voro force }=\frac{\cos (2 x)}{2}+0.5
$$

The CPD method uses a global Personal Space Map (PSM) to mask over the defined space and to accelerate the nearest entity search by carefully pointing and calculating its short-range directional vectors. The Voro force is used to compute the response to a local violation to personal space. In order to balance the local response against the intended global path, we used a Voro force that has to be specifically defined such that the resultant path has to be either 1 or 0 . Equation (1) represents this motion, and we integrated the equation into the net Voro force entity system to mimic a real life behavior, like the on observed in vehicles or objects. Here, the $\mathbf{x}$ in equation (1) is the angle between the local Voro force and the global path vector.

The entities are modeled after this governing force in the form of a linear force in the direction of the centroid. Based on this approach, personal space uses a geometrical approach. It is computed over the entire defined scene of simulated obstacles, vehicles, and entities, as seen in figure 5 . The resulting force $\mathbf{f}$ is formed from repelling the force of the personal space, denoted as the penalty force $\mathbf{p}$, and the attraction to 
the destination global force $\mathrm{g}$. This calculated approach represents individuality, and it opens the possibility of parallel computing. This, in particular, is exploited for better acceleration of calculation and load sharing in GPUs or multicore CPUs.

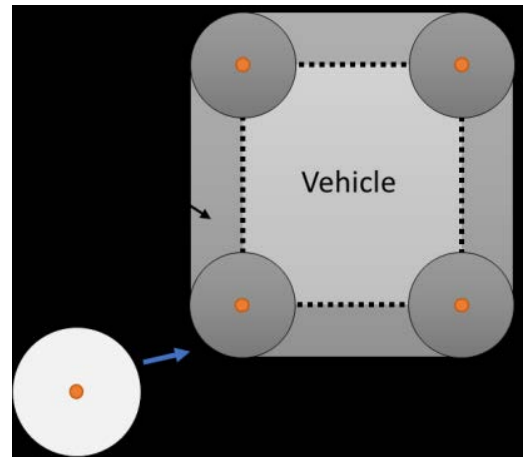

(a)

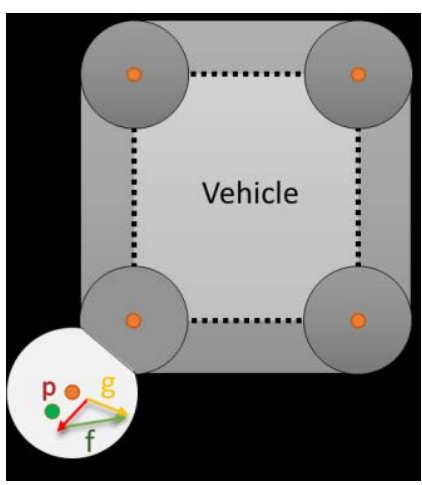

(c)

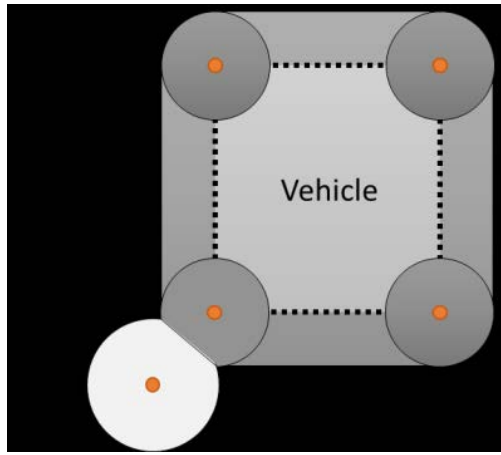

(b)

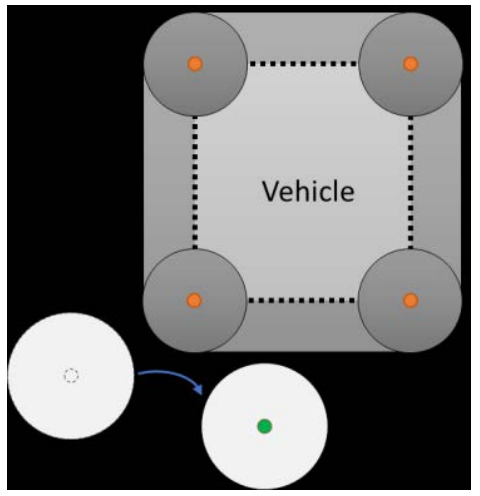

(d)

Figure 5. Representation of an entity experiencing a net force $f$ from global path force $g$ and Collison penalty force $p$.

Figure 6 shows an example that shows the interaction between heterogeneous entities like the ones we just presented. This simulation scenario uses a minimal number of entities, and it shows the behavior of the entities and evaluates their interaction with each other. To experiment includes three vehicles and an entity that is trying to cross the path from bottom to top. The vehicles have no destination or desired path. Vehicles are self-propelled on their own in their desired straight path. We used the least squares algorithm to predict the next location and restrict movement, and also changed the center of gravity of each vehicle in the object files itself, as this unwanted moment is the source of irregular centroidal points. Changing each object's centroid is necessary to make the vehicles stand still. 

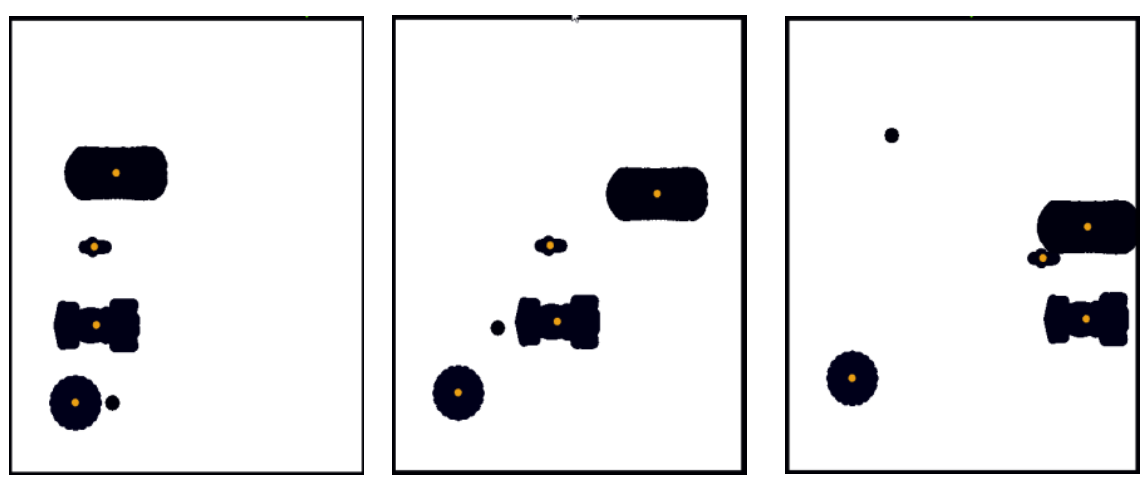

Figure 6. Objects displaying self-moving behavior in the scene without designated destinations.

\section{CASE STUDIES}

In this section we show the simulation results of various case studies with pedestrians, vehicles, and heterogeneous scenarios. Each simulation is run with fixed personal space radii ranging from 7 to 8 pixels to achieve the $0.8 \mathrm{~m}$ ideal personal space radius. The crowds are positioned randomly across the scene for each simulation. An ideal case of all adults was assumed in each scenario along with equal personal space in the simulation scene.

The first scenario shows a Motorcycle trying to pass through a dense crowd. In this scenario, a motorcycle is trying to pass through a dense crowd count of 100 from left to right while the half of the crowds are moving from bottom to top and the remaining 50 entities are trying to move from top to bottom.

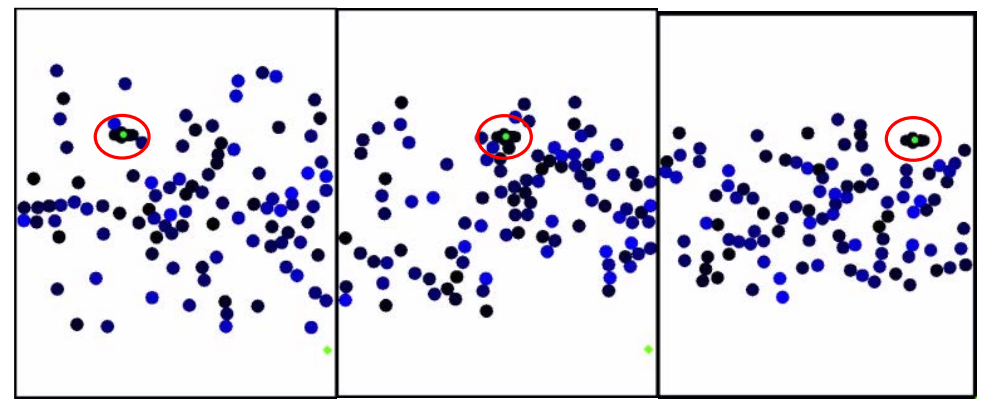

Figure 7. Motorcycle crossing through crowd moving in a bipolar direction.

We conducted various simulation scenarios discussed in Table 1. After certain crowd count the vehicle moment duration from source to destination remains close to constant; the reason for this is the size of the crowd density around the moving vehicle.

Table 1. Time taken for the Motorcycle to cross path depending on crowd count.

\begin{tabular}{|c|c|}
\hline Crowd count & Average time consumed to reach the destination \\
\hline 100 & 9.90 seconds \\
\hline 300 & 12.28 seconds \\
\hline 500 & 15.54 seconds \\
\hline 900 & 15.91 seconds \\
\hline
\end{tabular}

In the next set of experiments, an ambulance is trying to cross the path from left to right through entities moving in a bipolar direction. In Figure 8, we can observe a tail side empty space. As in real-world 
scenarios, the crowd do not have any option to step aside from an ambulance that is passing. When this happens, we can notice that people do not tend to go behind the back of ambulance just because of available empty space. We can notice such emergent behavior in the simulation justifying the assumed geometrically defined forces and parameters for the simulation.
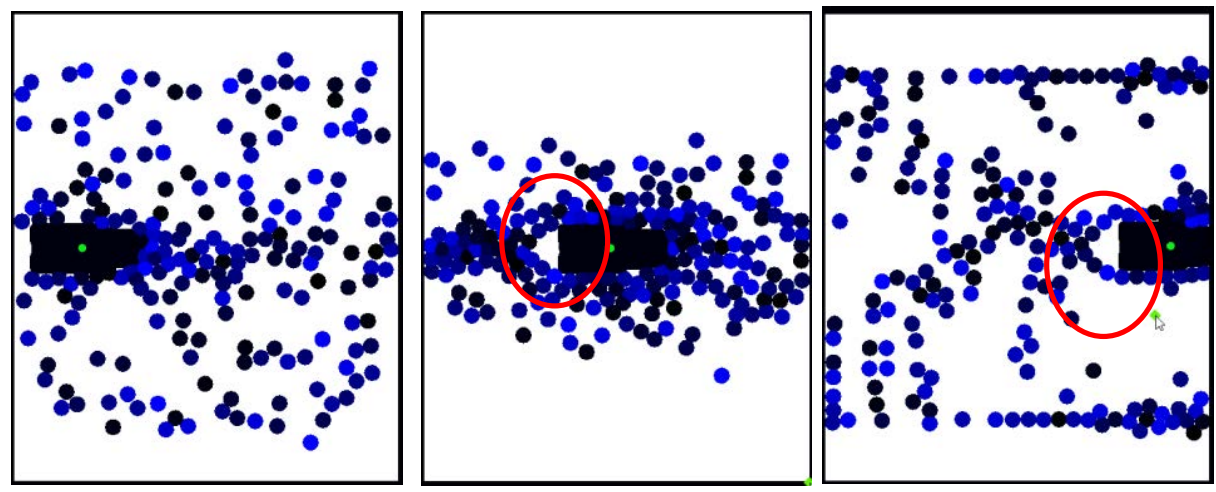

Figure 8. Ambulance trying to pass through 200 entities; tail space caused due to the moment.
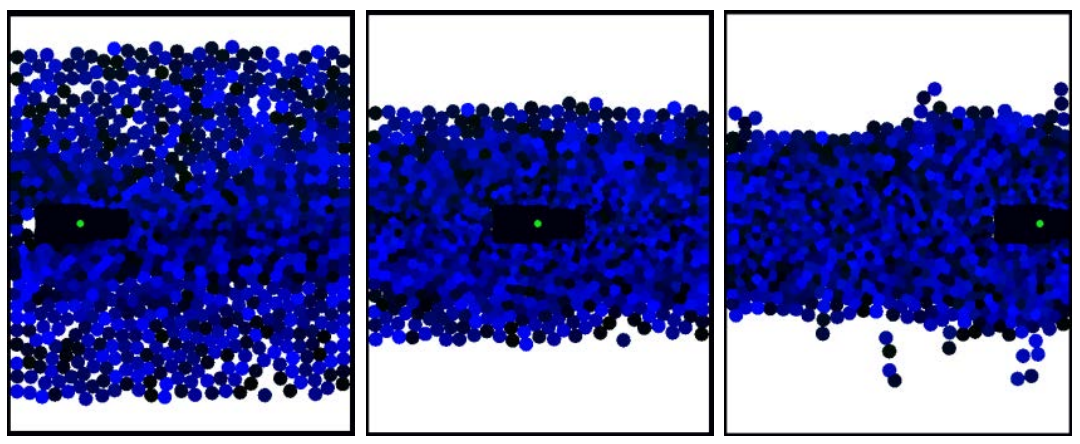

Figure 9. Simulation with an ambulance trying to pass through 900 entities.

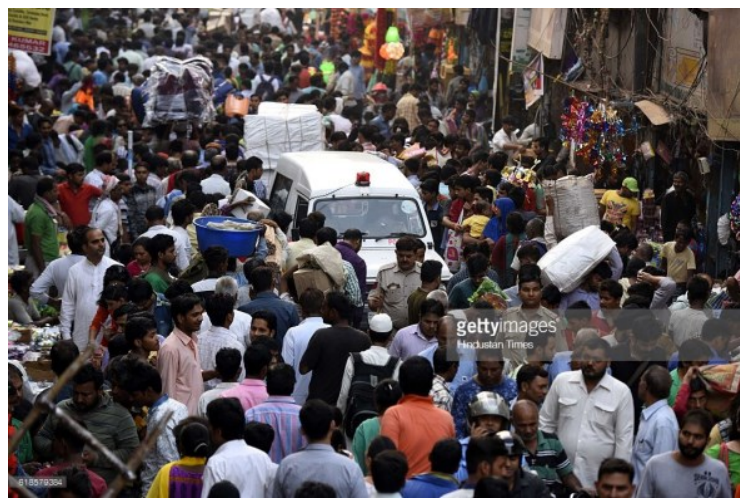

Figure 10. An ambulance trying to pass through the dense crowd.

In the next experiment, an ambulance is trying to cross the path by following a lane from bottom to top through the crowd that is standstill and uncooperative. This simulation is run with 300 entities with previously defined global parameters except for the standstill setting. The ambulance took 15.4 seconds to cross by following the designated lane. 

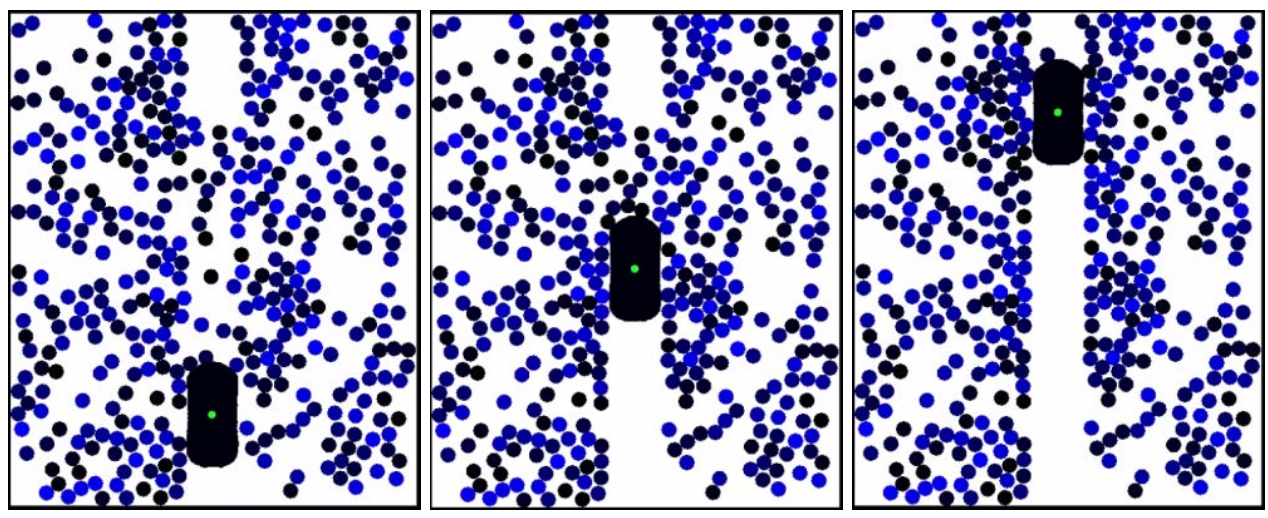

Figure 11. A simulation where an ambulance trying to pass through 400 uncooperative entities in a lane.

This simulation was run multiple times to get an averaged values of the vehicles crossing times. We changed the crowd count varying from 100 to 900 entities representing in the same scene, this increase in crowd count is used in this case to simulate dense crowd situations and behavior among the crowd under high reduction of personal space and the unwanted introduction of vehicle to the scene.

Table 2. Amount of time taken for the ambulance to cross path depending on crowd count.

\begin{tabular}{|c|c|}
\hline Crow count & Average time consumed to reach the destination \\
\hline 100 & 10.19 seconds \\
\hline 300 & 15.4 seconds \\
\hline 500 & 22.94 seconds \\
\hline 900 & 37.7 seconds \\
\hline
\end{tabular}

The experiment shown in Figure 12 considers five vehicles in a two-lane space. The crowd is equally introduced in one-third quadrant from the left side and the right side one-third quadrant with 400 crowd count. The dispersion of crowd is randomized on every simulation run. The vehicle movement is fixed from south to north with individually designated destination positions. To simulate crowd interaction with moving traffic, there are barricades on both sides of the vehicles to simulate a road environment.

The simulation demonstrates how the crowd interacts with a moving traffic, representing a truly heterogeneous environment. The barricades are forcing the crowd to take a detour and causing a stream of individuals. The entities are colliding with the vehicles personal space and altering their directional vector path as per geometrically defined voro force. Though the net force of combined entities is overwhelming the vehicle, the vehicle prefers to keep its destination vector and choose to ignore in recalculating its resultant destination path. Moreover, the entities are in a free flow and choose to favor the resultant Voro force derived from resulting vectors. This experimental simulation of such scenario is visually appealing and mimicking a real life like situation. This opens up the possibility of future opportunities in creating a multi-lane traffic junctions and traffic simulations that are highly customizable and scalable. 


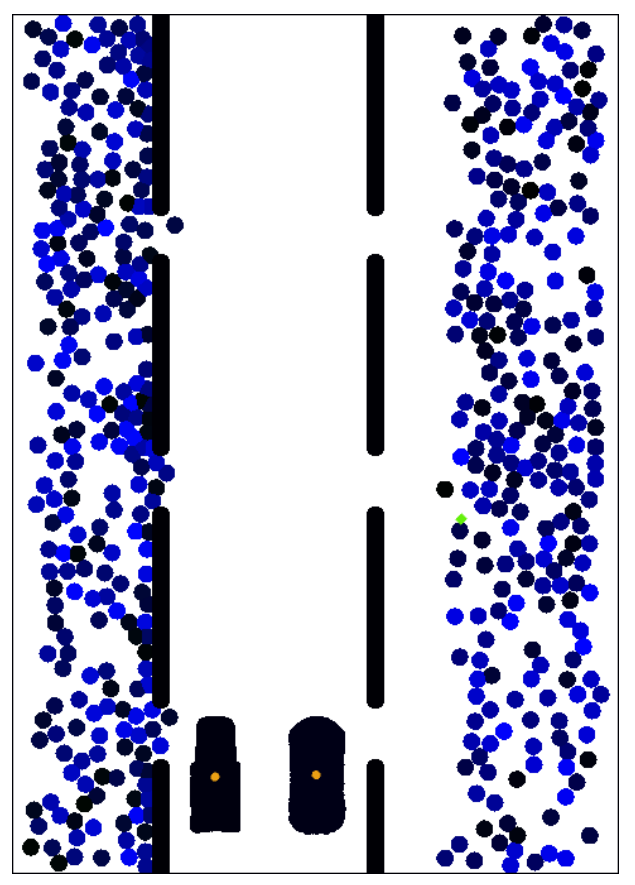

(a)

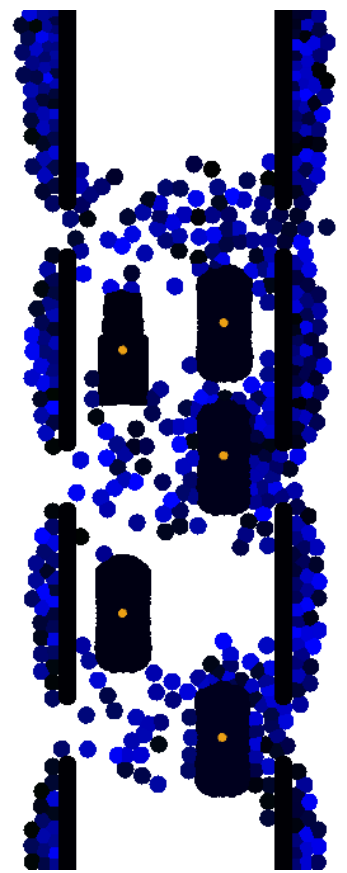

(c)

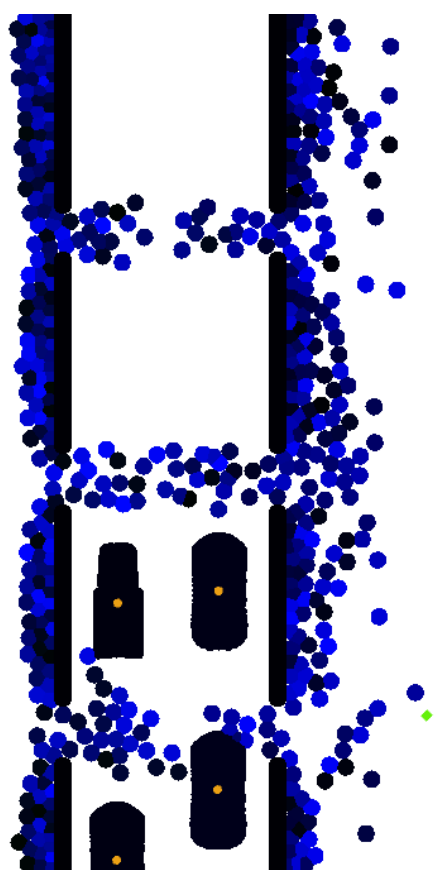

(b)

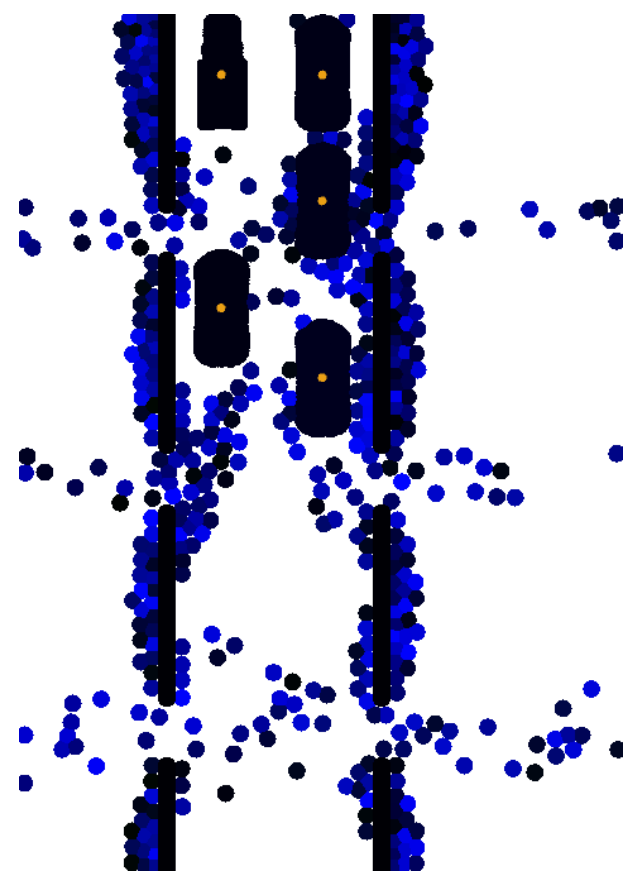

(d)

Figure 13. Two lane vehicles interaction with bipolar crowd movement.

\section{CONCLUSION}

We introduced an extension to the CPD model for crowd modelling and simulation that addresses the addition of vehicles, Motorcycles, obstacles entities into a single system; making it heterogeneous. We added the Minkowski sum approach, flexibility for adding foreign objects and simulating traffic scenarios. The developed engine displays potential to simulate multiple scenarios of vehicle and crowd interactions. 
We made use of some of such scenarios and reviewed in-depth analysis on each case while keeping the global variables fixed as per defined geometrically derived variables and global path parameters. The tool used in simulating has the potential for prototyping interactive simulations and cross-platform flexibility.

From the simulation results, the vehicles are leaving a trail of empty space upon forward movement which resembles a real-life scenario. Likewise, the simulation results show that upon increasing the density of the crowd, the vehicle moment is restricted. Based on this result we can determine which are the upper limits of the crowd density that will lead to a traffic jam situation. For future development, gathering more statistical data from real life events or social experiments will greatly improve the behavioral patterns used in these heterogeneous simulations.

\section{REFERENCES}

Andrade, E. L., Blunsden, S., Fisher, R. B. 2006. "Modelling crowd scenes for event detection". International Conference on Pattern Recognition, Hong Kong, China.

Cao, T., Wu, X., Guo, J., Yu, S., Xu, Y. 2009. "Abnormal crowd motion analysis". In 2009 IEEE International Conference on Robotics and Biomimetics. Guilin, China.

Fabeković, Z., Eškinja, Z., Vukić, Z. 2007. "Micro ROV Simulator". Proceedings of Elmar - International Symposium Electronics in Marine. Zadar, Croatia.

Hesham, O., Wainer, G. 2017. "Context-sensitive Personal Space for Dense Crowd Simulation". Symposium on Simulation in Architecture and Urban Design. Toronto, ON.

Huang, K., Zheng, X., Yang, Y., Wang, T. 2015. "Behavioral evolution in evacuation crowd based on heterogeneous rationality of small groups". Appl. Math. Comput., vol. 266, pp. 501-506.

Ivancevic, V. G.; Reid, D. J.; Aidman, E. V. 2010. "Crowd behavior dynamics: Entropic path-integral model”. Nonlinear Dynamics, vol. 59, no. 1-2, pp. 351-373.

Kullu, K., Güdükbay, U., Manocha, D. 2017. "ACMICS: an agent communication model for interacting crowd simulation”. Autonomous Agents and Multi-Agent Systems. Vol. 31, no. 6, pp. 1403-1423.

Lien, J. M. 2007. "Point-based Minkowski sum boundary". Proceedings of the Pacific Conference on Computer Graphics and Applications. Maui, HI.

Lister W. D., Day, A. 2012. "Stream-based animation of real-time crowd scenes". Computers and Graphics vol. 36 , no. 6, pp. 651-657.

Moussaid, M., Helbing, D., Theraulaz, G. 2011. "How simple rules determine pedestrian behavior and crowd disasters". Proceedings of the National Academy of Sciences.108:17, pp. 6884-6888.

Narain, R., Golas, A., Curtis, S., Lin, M. C. 2009. "Aggregate dynamics for dense crowd simulation". ACM SIGGRAPH Asia 2009. Yokohama, Japan.

Patil, S., Van Den Berg, J., Curtis, S., Lin, M. C., Manocha, D. 2011. "Directing Crowd Simulations Using Navigation Fields". IEEE Transactions on Visualization and Computer Graphics. 17:2, pp. 244-254.

Pettré, J. Ondřej, J., Olivier, A.-H, Cretual, A., Donikian, S. 2009. "Experiment-based modeling, simulation and validation of interactions between virtual walkers". Proceedings of the 2009 ACM SIGGRAPH/Eurographics Symposium on Computer Animation. Rennes, France.

Pustylnik G., Sharir, M. 2003. "The Minkowski sum of a simple polygon and a segment". Information Processing Letters, vol. 85, no. 4, pp. 179-184. 
Rangel, A., Ballinas, A. L.; Muñoz, A. 2017. "An entropy model to measure heterogeneity of pedestrian crowds using self-propelled agents”. Physica A, 473, pp. 213-224.

Sarkar, P. 2000. “A brief history of cellular automata”. ACM Computer Surveys, 32:1, pp. 80-107.

Schiff, J. 2012. Cellular Automata: A Discrete View of the World. Wiley.

Zheng, L., Qin, D., Cheng, Y., Wang, L., Li, L. 2016. "Simulating heterogeneous crowds from a physiological perspective". Neurocomputing, vol. 172, pp. 180-188.

\section{AUTHOR BIOGRAPHIES}

SRIVASTAV JANAPALLI received a Bachelor in Electrical and Electronics Engineering at the BV Raju Institute of Technology (BVRIT) in 2013. He completed a Masters in Engineering at Carleton University in 2018. His research and projects center around adaptive learning, IoT, retail POS transaction trends and crowd simulations. His experience includes work in stock market transaction analysis, web app development and heterogeneous crowd simulations. His email address is SrivastavJanapalli@.cmail.carleton.ca.

OMAR HESHAM received a Bachelor of Information Technology (2009) a Master of Information Science (2012) and a PhD in Electrical Engineering (2019) at Carleton University. He obtained the Best Paper Award at SIMAUD 2016 and 2017. He currently is the Graphics and High-Performance Computing Lead at Zetane Systems, Montreal, Canada. His $\mathrm{PhD}$ research focused on agent-based simulation and visualization at Carleton University's Advanced Real-time Simulation Lab. His email address is omar.hesham@carleton.ca.

GABRIEL A. WAINER (FSCS, SMIEEE), received the M.Sc. (1993) at the University of Buenos Aires, Argentina, and the Ph.D. (1998, with highest honors) at the Université d'Aix-Marseille III, France. In 2000 he joined the Department of Systems and Computer Engineering at Carleton University (Ottawa, ON, Canada), where he is now Full Professor and Associate Chair for Graduate Studies. He is the author of three books and numerous research articles; he edited four other books, and helped organizing numerous conferences, including being one of the founders of the Symposium on Theory of Modeling and Simulation, SIMUTools and SimAUD. Prof. Wainer was Vice-President Conferences and Vice-President Publications and is a member of the Board of Directors of the SCS. Prof. Wainer is the Special Issues Editor of SIMULATION, member of the Editorial Board of IEEE Computing in Science and Engineering, Wireless Networks (Elsevier), Journal of Defense Modeling and Simulation (SCS). He is the head of the Advanced Real-Time Simulation lab, located at Carleton University's Centre for advanced Simulation and Visualization (V-Sim). He has been the recipient of various awards, including the IBM Eclipse Innovation Award, SCS Leadership Award, and various Best Paper awards. He has been awarded Carleton University's Research Achievement Award (2005, 2014), the First Bernard P. Zeigler DEVS Modeling and Simulation Award, the SCS Outstanding Professional Award (2011), Carleton University's Mentorship Award (2013), the SCS Distinguished Professional Award (2013), and the SCS Distinguished Service Award (2015). He is a Fellow of SCS. His e-mail address is gwainer@sce.carleton.ca. 